\title{
ON THE ZEROS OF TOTAL SETS OF POLYNOMIALS
}

\author{
BY \\ PHILIP DAVIS AND HENRY POLLAK
}

1. Introduction. In the plane of the complex variable $z=x+i y$, we shall deal with domains $B$ which are schlicht, bounded, and simply-connected. Let $L_{B}$ designate a class of functions which are regular in $B$ and which possess the additional property that to every boundary point $z_{b}$ of $B$ and to every $\epsilon$, there exist functions of the class with a singularity in the circle $C\left(z_{b} ; \epsilon\right)$ : $\left|z-z_{b}\right|<\epsilon$. As examples of such classes, let us note

(a) the class of all functions regular in $B$,

(b) the class consisting of a single function which possesses the boundary of $B$ as its natural boundary.

A set of polynomials

$$
\begin{aligned}
p_{n}(z)=\sum_{k=0}^{n} a_{n k} z^{k}=a_{n n}\left(z-z_{1}^{(n)}\right) \cdots & \left(z-z_{n}^{(n)}\right), \\
& a_{n n} \neq 0(n=0,1,2, \cdots),
\end{aligned}
$$

will be said to be total over $L_{B}$ if

(a) the zeros of the polynomials are bounded:

$$
\left|z_{i}^{(n)}\right| \leqq M \quad(n=1,2,3, \cdots ; i=1,2, \cdots, n),
$$

and if

(b) every function of the set $L_{B}$ possesses an expansion of the form

$$
f(z)=\sum_{n=0}^{\infty} b_{n} p_{n}(z)
$$

convergent at every interior point of $B$.

We shall mention four examples of total sets.

(a) The set of polynomials orthonormalized over the boundary of $B$ (Szegö [9]):

$$
\int_{b} p_{m}(z)\left(p_{n}(z)\right)^{-} d s=\delta_{m n} ; \quad \operatorname{Re}\left(a_{n n}\right)>0 .
$$

(b) The set of polynomials orthonormalized over the area $B$ (Bergman [1], Carleman [3]):

$$
\iint_{B} p_{m}(z)\left(p_{n}(z)\right)-d x d y=\delta_{m n} ; \quad \operatorname{Re}\left(a_{n n}\right)>0 .
$$

Received by the editors February 20, 1951 and, in revised form, April 16, 1951. 
With some additional restrictions as to the nature of the boundary of $B$, the set (4) is known to be total over the set of functions regular in and on the boundary of $B$, while the set (5) is total over the set of functions $f$ which are regular in $B$ and such that $\iint_{B}|f|^{2} d x d y<\infty$. The zeros of these polynomials lie in the convex hull of $B$. In both cases, weight functions may be supplied.

(c) Let $z_{1}, z_{2}, \cdots, z_{k}$ be $k$ distinct complex points. Define polynomials $p_{n}(z)$ as follows:

$$
\begin{aligned}
p_{0}(z) & =1 \\
p_{1}(z) & =z-z_{1}, \\
\cdots & \cdots \cdot \\
p_{k}(z) & =\left(z-z_{1}\right)\left(z-z_{2}\right) \cdots\left(z-z_{k}\right), \\
p_{k+1}(z) & =\left(z-z_{1}\right)\left(z-z_{2}\right) \cdots\left(z-z_{k}\right)\left(z-z_{1}\right),
\end{aligned}
$$

If, now, $B$ is the interior of (any loop of) the lemniscate

$$
L:\left|\left(z-z_{1}\right)\left(z-z_{2}\right) \cdots\left(z-z_{k}\right)\right|=c ; \quad c>0,
$$

then the set (6) will be total over the set of functions regular in the interior of $B$. (See, e.g., Walsh [12, p. 57].)

(d) As the fourth and final example, we shall mention the Faber polynomials for a domain (Faber [4]). Let the function

$$
w=\phi(z)=z+a_{0}+\frac{a_{1}}{z}+\frac{a_{2}}{z^{2}}+\cdots
$$

map the exterior of the domain $B$ onto the exterior of the circle $|w|=a$, $a>0$. The Faber polynomials are defined as

$$
p_{n}(z)=\text { principal part of }[\phi(z)]^{n} \quad(n=0,1, \cdots) .
$$

They are known to be total over the set of functions regular in $B$.

In our first theorem, we relate the derived set of the set of zeros of a total set of polynomials with the geometry of the domain. Here, it is shown that the limit points of zeros cannot lie exclusively in any "half" of the domain. A variety of applications has been found for this theorem. It has been used in Corollary 1.2 to obtain geometric information about lemniscates, and in Theorem 2 to prove the existence at certain nondifferentiable portions of the boundary of $B$ of limit points of the zeros of total sets. In $\$ 4$, an application is made, through the Faber polynomials, to conformal mapping; this yields theorems on the possible analytic continuation of the mapping functions of a domain with an analytic boundary as well as distortion theorems for schlicht functions of a type obtained by Golusin. Applications to the theory of overconvergence and to theorems of Jentzsch type are also possible, but have not 
been discussed here. In $\S 3$, the assumption is made that the zeros of a total set possess a density. It is found that in this case, the domain $B$ over which the set is total cannot be arbitrary, and, indeed, must have as boundary a level line of a logarithmic potential.

In $\$ 5$, several of the results of $\$ 4$ have been generalized to the case of multiply-connected domains. At first, a theorem analogous to Theorem 1 is proved for total sets of rational functions. This theorem is then applied to certain specific sets of rational functions introduced by Walsh, and yields theorems on the possible analytic continuation of the interior Green's function of a domain, and of various harmonic measures relevant to the domain. These theorems are purely geometric in character.

2. On the derived set of the set of zeros.

THEOREM 1. Let $z_{b}$ and $z_{b^{\prime}}$ designate two distinct points lying on the boundary of a domain $B$. Then the half-planes

$$
\begin{aligned}
& \left|z-z_{b}\right| \geqq\left|z-z_{b^{\prime}}\right|, \\
& \left|z-z_{b^{\prime}}\right| \geqq\left|z-z_{b}\right|
\end{aligned}
$$

must each contain a limit point of zeros of the polynomials of any set which is total over an $L_{B}$.

Proof. Let $z_{I}$ designate a point which is interior to $B$. This point will be thought of as being fixed initially. For a value $t$ with $0<t<1$, the set of all points $z$ satisfying

$$
\left|\frac{z-z_{b}}{z-z_{I}}\right| \leqq t
$$

lie in or on a circle of Apollonius $A\left(z_{b}, z_{I} ; t\right)$ whose "foci" are at $z_{b}$ and $z_{I}$, that circle being selected which contains $z_{b}$ in its interior. We shall show firstly that for all fixed $t, 0<t<1$, an infinite number of zeros $z_{i}^{(n)}$ must lie in comp $A$. Select a $t^{\prime}$ with $0<t<t^{\prime}<1$, and define a $\delta$ by

$$
0<\delta<\min \left\{\frac{1}{2}\left(t^{\prime}-t\right)\left|z_{b}-z_{I}\right|,\left(\frac{t}{1+t}\right)\left|z_{b}-z_{I}\right|\right\} .
$$

The circle $C\left(z_{b} ; \delta\right)$ will then be contained in the circle $A$. Suppose now that an infinite number of zeros do not lie in comp $A$. Then, for all $n \geqq n_{0}, z_{k}^{(n)}$ lie in $A$. If, therefore, $z \in C\left(z_{b} ; \delta\right)$, we have

$$
\begin{gathered}
\frac{\left|z-z_{k}^{(n)}\right|}{\left|z_{I}-z_{k}^{(n)}\right|} \leqq \\
\frac{\left|z-z_{b}\right|}{\left|z_{I}-z_{k}^{(n)}\right|}+\frac{\left|z_{b}-z_{k}^{(n)}\right|}{\left|z_{I}-z_{k}^{(n)}\right|} \leqq \frac{\delta}{\left|z_{I}-z_{k}^{(n)}\right|}+t, \\
\frac{\delta}{1 / 2\left|z_{I}-z_{b}\right|}+t<t^{\prime}<1 \quad\left(n \geqq n_{0}\right) .
\end{gathered}
$$


Let a function of the class $L_{B}$ now be selected for which there is a singularity in the circle $C\left(z_{b} ; \delta\right)$. Such a function must exist and will possess an expansion

$$
f(z)=\sum_{n=0}^{\infty} b_{n} p_{n}(z)=\sum_{n=0}^{\infty} b_{n}^{\prime}\left(z-z_{1}^{(n)}\right) \cdots\left(z-z_{n}^{(n)}\right),
$$

converging for all interior points. In particular, it converges for $z=z_{I}$, and therefore for some $M>0$ and for all $n \geqq n_{1}$,

$$
\left|b_{n}^{\prime}\right| \leqq M\left\{\left|\left(z_{I}-z_{1}^{(n)}\right) \cdots\left(z_{I}-z_{n}^{(n)}\right)\right|\right\}^{-1} .
$$

The claim is now made that the series (14) converges uniformly and absolutely for all $z \in C\left(z_{b} ; \delta\right)$. For,

$$
\begin{aligned}
\sum_{n=n_{1}}^{\infty} b_{n}^{\prime}\left(z-z_{1}^{(n)}\right) \cdots\left(z-z_{n}^{(n)}\right) & \ll M \sum_{n=n_{1}}^{\infty} \frac{\left|z-z_{1}^{(n)}\right| \cdots\left|z-z_{n}^{(n)}\right|}{\left|z_{I}-z_{1}^{(n)}\right| \cdots\left|z_{I}-z_{n}^{(n)}\right|} \\
& <M \sum_{n=n_{1}}^{\infty} t^{\prime n}<\infty .
\end{aligned}
$$

Thus in $C\left(z_{b} ; \epsilon\right)$ the series $\sum_{n=0}^{\infty} b_{n} p_{n}(z)$ is a regular function $f_{1}(z)$, while in $B \cap C\left(z_{b} ; \epsilon\right)$ it coincides with the regular function $f(z)$. Therefore, $f_{1}(z)$ is an analytic continuation of $f(z)$ to $C\left(z_{b} ; \boldsymbol{\epsilon}\right)$. This is impossible because of the singularity of $f(z)$ in this circle.

We next show that for fixed $z_{b}$ and $z_{I}$, the half-plane

$$
\left|z-z_{b}\right| \geqq\left|z-z_{I}\right|
$$

must contain a limit point of zeros. Select a sequence $t_{1}<t_{2}<\cdots$, with $\lim _{n \rightarrow \infty} t_{n}=1$. It is clear that the point set

$$
\bigcap_{n=1}^{\infty} \operatorname{comp} A\left(z_{b}, z_{I} ; t_{n}\right)
$$

is the half-plane (17). The result now follows, inasmuch as the zeros are assumed to be bounded. If no assumption of boundedness had been made, we could only conclude that there are an infinite number of zeros in each region comp $A\left(z_{b}, z_{I} ; t\right)$. Finally, to arrive at (10a), select a sequence of interior points $z_{I_{n}}$ with $\lim _{n \rightarrow \infty} z_{I_{n}}=z_{b^{\prime}}$. (10b) follows by symmetry.

It should be noted that the half-planes (10a) and (10b) are bounded by the perpendicular bisector of the chord $\left[z_{b} z_{b^{\prime}}\right]$. By allowing $z_{b^{\prime}} \rightarrow z_{b}$, we see that Theorem 1 also holds for the half-planes bounded by the normals to the boundary of $B$. It will be convenient to adopt a notation for the family of all normals and perpendicular bisectors of chords of a given domain. If the given domain has boundary $C$, we shall designate this family by $N(C)$.

COROLlaRY 1.1. Let the zeros of a set of polynomials total over $L_{B}$ possess a 
single limit point. Then $B$ must necessarily be a circle.

For in this case, the perpendicular bisectors of all chords must pass through the single limit point. The figure is therefore a circle.

COROLlaRy 1.2( $\left.{ }^{1}\right)$. The normals and the perpendicular bisectors of all chords of (any loop of) the lemniscate (7) must meet the convex hull of the foci $z_{1}, z_{2}, \cdots, z_{k}$.

For the set (6) is total over the set of functions regular in the interior of the lemniscate, and the foci are the only limit points of the zeros.

By employing any set of Jacobi polynomials $P_{n}^{(\alpha, \beta)}(z)$ (cf. Szegö $[10, p$. 238]) we may obtain a similar and elementary result for ellipses: if $C$ designates an arbitrary ellipse, then each line of the family $N(C)$ must intersect the focal segment $F F^{\prime}$. But more generally, we have the following:

COROLlaRY 1.3. Let $\left\{p_{n}(x)\right\}$ be any set of polynomials which are orthonormal over $(-1,1)$ with respect to $\alpha(x)$ : i.e.,

$$
\int_{-1}^{+1} p_{n}(x) p_{m}(x) d \alpha(x)=\delta_{m n} \quad(m, n=0,1,2,3, \cdots) .
$$

$\alpha(x)$ may be selected as any nondecreasing function which possesses infinitely many points of increase and such that

$$
\int_{-1}^{+1} d \alpha(x)<\infty
$$

Then the set $\left\{p_{n}(z)\right\}$ can be total only over domains whose boundary $C$ is such that each line of the family $N(C)$ intersects the segment $(-1,1)$.

Proof. In this case (see, e.g., Szegö [9, p. 43]), the zeros of the set are confined to the segment $(-1,1)$. If a line of the family $N(C)$ did not intersect $(-1,1)$, the conclusion of Theorem 1 would be contradicted.

In Whittaker [14], the notion of a basic series of polynomials effective for a domain $B$ is defined and studied. Briefly, a set of polynomials is said to be effective in $B$ if each function regular in $B$ has an expansion (3) uniformly convergent in every closed subdomain. The coefficients $b_{n}$ are to be determined by applying to $f$ a sequence of operators which are essentially linear differential operators of infinite order. A basic set of polynomials $p_{n}(z)=z^{n}+\cdots$ which is effective for $B$ will be total over $B$ in our sense if the set of zeros is bounded. Thus, theorems on the effectiveness of basic series may yield,

(1) Cf. Walsh [11] where a similar theorem has been proved for the normals. Walsh uses potential theory to arrive at this result for the lemniscate, and, more generally, for the level curves of the Green's function of the domain exterior to a closed boundary set. Corollary 1.2 may be generalized by utilizing the Newton interpolation polynomials whose distribution of zeros is more general than that of (6). In this connection, see Goncharov [6]. Cf. also Theorem 7. 
through Theorem 1, statements about the distribution of their zeros. For example, we have the following.

COROllary 1.4. Let $p_{n}(z)$ be given by (1) with the coefficients $a_{n k}$ subject to $\left|a_{n k}\right| \leqq M \sigma^{n-k}(k=0,1, \cdots, n ; n=0,1, \cdots), a_{n n}=1$. Then the set $\left\{p_{n}(z)\right\}$ has a limit point in each closed half-plane $\operatorname{Re}\left(e^{i \theta} z\right) \geqq 0,0 \leqq \theta \leqq 2 \pi$.

Proof. Under the above restriction, the set of polynomials is known to be effective in a circle $|z|<T$, for $T$ sufficiently large (Boas [2]). Now the zeros of the set are bounded. For, let $z_{k}^{(n)}$ be a zero of $p_{n}(z)$. Then,

$$
\begin{aligned}
\left|z_{k}^{(n)}\right|^{n} & \leqq\left|a_{n, n-1}\right|\left|z_{k}^{(n)}\right|^{n-1}+\cdots+\left|a_{n 0}\right| \\
& \leqq M\left|z_{k}^{(n)}\right|^{n-1} \sigma+M\left|z_{k}^{(n)}\right|^{n-2} \sigma^{2}+\cdots+M \sigma^{n} .
\end{aligned}
$$

Thus,

$$
\frac{1}{M} \leqq\left|\frac{\sigma}{z_{k}^{(n)}}\right|+\cdots+\left|\frac{\sigma}{z_{k}^{(n)}}\right|^{n}
$$

If, now, $z_{k}^{(n)} \rightarrow \infty(n \rightarrow \infty)$, then, ultimately, $\left|\sigma / z_{k}^{(n)}\right|<1$, so that

$$
\frac{1}{M} \leqq \frac{\left|\sigma / z_{k}^{(n)}\right|^{n+1}}{1-\left|\sigma / z_{k}^{(n)}\right|}=o(1)
$$$$
(n \rightarrow \infty) \text {. }
$$

This is impossible. Thus, the set of polynomials is total over $|z|<T$, and the conclusion follows.

CoROllary 1.5. Let the limit points of the zeros of $p_{n}(z)$ be bounded and possess a closed convex hull which does not contain the origin. Then the set cannot be total (hence a fortiori effective) over any circle $|z|<R$.

By choosing $z_{b}$ and $z_{b^{\prime}}$ in Theorem 1 as widely separated points, it appears that the limit points of zeros of a total set cannot lie exclusively in any "half" of the domain. By choosing $z_{b}$ and $z_{b^{\prime}}$ sufficiently close together along a portion of smooth arc, it follows, as we have already observed, that a limit point of zeros must be located on either side of a normal to the boundary, the normal itself being included. At nondifferentiable portions of the boundary, an interesting phenomenon may appear. We shall describe this in our next theorem and show by this means that domains exist for which certain total sets must necessarily have limit points of zeros on the boundary.

THEOREM 2. Let the zeros of a total set over an $L_{B}$ be contained in a domain $B^{*}$. Let $z_{b_{1}}^{(n)}, z_{b_{2}}^{(n)}$ be two sequences of boundary points of $B$. Designate by $P^{(n)}$ the corresponding sequence of half-planes

$$
\left|z-z_{b_{1}}^{(n)}\right| \leqq\left|z-z_{b_{2}}^{(n)}\right|
$$


If $z_{b}$ is a boundary point of $B$ for which, in the point set sense, we have

$$
z_{b}=\bigcap_{n=1}^{\infty}\left[P^{(n)} \cap B^{*}\right],
$$

then $z_{b}$ is a limit point of zeros.

Proof. By Theorem 1, each $P^{(n)} \cap B^{*}$ contains a limit point of zeros. If the circle $C\left(z_{b} ; \epsilon\right)$ be drawn, then, for $n$ sufficiently large, $P^{(n)} \cap B^{*}$ is contained in this circle. Thus, there must be an infinite number of zeros in each $C\left(z_{b} ; \epsilon\right)$.

As a special case, we have then the following result:

CoRollary 2.1. If the domain $B$ is convex and possesses at $z_{b}$ a corner whose angle is less than $\pi / 2$, then $z_{b}$ is a limit point of the zeros of the orthonormal polynomials (4) and (5) (or any total set whose zeros lie in B).

Proof. In this case, we may select $B^{*}=B$. Now choose $z_{b_{1}}(n) \equiv z_{b}$, and $z_{b_{2}}(n)$ as a sequence of boundary points approaching $z_{b}$ on one side of the corner. The condition (18) is clearly satisfied.

We may employ Theorem 2 to give an example showing that if the boundary of a domain is deformed continuously, the motion of the zeros of the orthonormal polynomials (4), (5) may, in a certain sense, be discontinuous. To show this, proceed as follows. Upon a small arc of the unit circle $U$ as a base, erect a triangle $T_{\epsilon}$, one of whose sides is a segment of length $\epsilon$ lying on an extension of a radius and whose vertex angle has a fixed value $\alpha<\pi / 2$. The domain $U+T_{\epsilon}$ satisfies the conditions of Theorem 2. The vertex of $T_{\epsilon}$ is therefore a limit point of zeros. This is true for all $\epsilon$. Yet, as $\epsilon \rightarrow 0, U+T_{\epsilon}$ approaches $U$ uniformly, a figure the zeros of whose orthonormal polynomials are all located at the origin.

3. On the strength of limit points. From the results of the preceding section, it appears that a knowledge of the distribution of the zeros of a set of polynomials will yield necessary conditions to be satisfied by any region over which the set is total. In particular (cf. Corollary 1.1), if sufficiently strong hypotheses are made as to their distribution, then the possible regions of totality are essentially determined. This corollary is capable of wide generalization. To this end, and in order to characterize more closely the distribution of zeros at a limit point, it is convenient to introduce the notion of the strength of a limit point (cf. Korovkin [7]).

Let $N(z, \epsilon, n)$ designate the number of the points $z_{1}^{(n)}, \ldots, z_{n}^{(n)}$ which lie in the circle $C(z ; \epsilon)$. We shall say that $z$ is a limit point of positive strength if

$$
\liminf _{n \rightarrow \infty}(1 / n) N(z, \epsilon, n)=t(\epsilon)>t^{\prime}>0,
$$

for all $\epsilon>0$. This means, roughly, that $z$ is a limit point of positive strength 
if at least a fixed positive proportion of the zeros of each polynomial cluster about it. Consider also the following situation. Suppose that the zeros have a finite number of limit points $z_{1}, z_{2}, \cdots, z_{p}$. If for each $i, i=1,2, \cdots, p$, and for some

$$
\epsilon_{i}<\min \left|z_{j}-z_{k}\right| \quad(j, k=1,2, \cdots, p ; j \neq k),
$$

we have

$$
\lim _{n \rightarrow \infty}(1 / n) N\left(z_{i}, \epsilon_{i}, n\right)=\rho_{i}>0 \quad(i=1,2, \cdots, p),
$$

then (20) will persist for all smaller $\epsilon_{i}$ and the result will therefore be independent of $\epsilon_{i}$. In such a case, we say that $z_{i}$ is a limit point of strength $\rho_{i}$.

Let the set $\left\{p_{n}(z)\right\}$ be total over an $L_{B}$. Each $f \in L_{B}$ therefore possesses an expansion of the form.

$$
f(z)=\sum_{n=0}^{\infty} b_{n}^{\prime}\left(z-z_{1}^{(n)}\right) \cdots\left(z-z_{n}^{(n)}\right),
$$

valid in $B$. We shall impose the further condition that

$$
\limsup _{n \rightarrow \infty}\left(\left|b_{n}^{\prime}\right|\right)^{1 / n} \leqq r<\infty ; \quad f \in L_{B},
$$

where $r$ is independent of the function $f$. Condition (22) is fulfilled for many sets. In particular, it is fulfilled for the sets (4), (5), (6), (9). It is fulfilled, in addition, for any total set whose zeros are not everywhere dense in $B$.

THEOREM 3. Let condition (22) be fulfilled. Then no boundary point $z_{b}$ can be a limit point of positive strength.

Proof. Assume the contrary. Then given an $\epsilon>0$, there will therefore exist an $n_{0}(\epsilon)$ such that for $n \geqq n_{0}(\epsilon), N\left(z_{b}, \epsilon, n\right) \geqq n t^{\prime}$. Hence for $\left|z-z_{b}\right| \leqq \epsilon$ and for $n \geqq n_{0}(\epsilon)$,

$$
\begin{aligned}
\left|z-z_{1}^{(n)}\right|\left|z-z_{2}^{(n)}\right| \cdots\left|z-z_{n}^{(n)}\right| & \leqq(2 \epsilon)^{n t^{\prime}}(M+\epsilon)^{n-n t^{\prime}} \\
& =\left[(2 \epsilon)^{t^{\prime}}(M+\epsilon)^{1-t^{\prime}}\right]^{n} .
\end{aligned}
$$

Now let $\epsilon$ be selected so small that $r(2 \epsilon)^{t^{\prime}}(M+\epsilon)^{1-t^{\prime}}=r^{\prime}<1$. By the definition of the class $L_{B}$, we can now find a function $f$ of this class which possesses a singularity in the circle $C\left(z_{b} ; \epsilon\right)$. But since

$$
\left|b_{n}^{\prime}\left(z-z_{1}^{(n)}\right) \cdots\left(z-z_{n}^{(n)}\right)\right| \leqq r^{\prime n} \quad\left(n \geqq n_{1}\right),
$$

the series (21) will converge absolutely and uniformly in $C\left(z_{b} ; \epsilon\right)$ and will therefore provide an analytic continuation of $f$ to this circle. This is a contradiction.

A more general assumption is that the zeros $z^{(n)}$ possess a density defined 
as follows. Let the zeros (assumed to be bounded) lie in a rectangle $R$. Let $r=r\left(x_{1}, x_{2} ; y_{1}, y_{2}\right)$ designate the rectangle $x_{1} \leqq x<x_{2} ; y_{1} \leqq y<y_{2}$, and $N(n, r)$ the number of zeros of $p_{n}(z)$ which lie in $r$. Assume that for each $r$, the limit

$$
\lim _{n \rightarrow \infty} \frac{1}{n} N(n, r)=\rho(r)
$$

exists. Under these assumptions, the region of totality must be bounded by a level line of the logarithmic potential

$$
\int_{R} \log |z-w| d \rho_{w}(r)=\text { const., }
$$

the integral being understood in the Riemann-Stieltjes sense. We shall prove this only in the case of a finite number of limit points of strength $\rho_{i}$. The general case is obtained in a similar fashion. By way of a lemma, we employ the following theorem which is due to Korovkin [7].

THEOREM 4. Let the roots of the polynomials

$$
q_{n}(z)=\left(z-z_{1}^{(n)}\right) \cdots\left(z-z_{n}^{(n)}\right)
$$

be bounded and possess a finite number of limit points of strengths $\rho_{i}(i=1$, $2, \cdots, p)$. If a sequence $\left\{c_{n}\right\}$ be given for which

$$
\limsup _{n \rightarrow \infty}\left|c_{n}\right|^{1 / n}=m<\infty,
$$

then the series $\sum_{n=0}^{\infty} c_{n} q_{n}(z)$ converges uniformly and absolutely at each point $z$ for which

$$
\prod_{i=1}^{p}\left|z-z_{i}\right| \rho_{i}<1 / m .
$$

Moreover, if $z$ is not a limit point of zeros of $q_{n}(z)$, and if

$$
\prod_{i=1}^{p}\left|z-z_{i}\right| \rho_{i}>1 / m,
$$

then the series diverges.

TheOREM 5. Let the polynomials $q_{n}(z)$ be total over an $L_{B}$. If their zeros possess only a finite number of limits points $z_{1}, z_{2}, \cdots, z_{p}$, each of positive strength $\rho_{i}$, then $B$ is necessarily a lemniscate:

$$
L_{M}: \prod_{i=1}^{p}\left|z-z_{i}\right| \rho_{i}=\frac{1}{M} ; \quad 0<M<\infty .
$$

Proof. Let the point $z^{*} \in B$ and not coincide with any of the limit points 
$z_{i}$. We can find an $r$ and an $N$ such that the polynomials $q_{n}(z)$ possess no zeros in the circle $C\left(z^{*} ; r\right)$ for $n \geqq N$. Hence, for $n \geqq N,\left|q_{n}\left(z^{*}\right)\right| \geqq r^{n}$. If an $f \in L_{B}$ be selected, it will have an expansion $f(z)=\sum c_{n} q_{n}(z)$ convergent at $z=z^{*}$. Hence, for some constant $K,\left|c_{n} q_{n}\left(z^{*}\right)\right|<K$, and therefore $\left|c_{n}\right|<K / r^{n}$, $n \geqq N$. Thus, $\lim \sup _{n \rightarrow \infty}\left|c_{n}\right|^{1 / n} \leqq 1 / r$. Inasmuch as $r$ is independent of the particular $f \in L_{B}$ chosen, $\lim \sup _{n \rightarrow \infty}\left|c_{n}(f)\right|^{1 / n}$ has a uniform upper bound for all $f \in L_{B}$, and hence a least upper bound. We shall designate this least upper bound by $M$.

We shall now show that $B=L_{M}$. In the first place, for every $f \in L_{B}$, $\lim \sup _{n \rightarrow \infty}\left|c_{n}(f)\right|^{1 / n} \leqq M$. Therefore, by Theorem 4 , every such $f$ is analytic in at least $L_{M}$. But since the class of functions $L_{B}$ contains functions with singularities arbitrarily close to the boundary of $B$, it follows that $L_{M}$ must be contained in $B$. In the second place, by the definition of $M$, we can find, for each positive $\epsilon$, a function $f_{\epsilon}$, such that $\lim \sup _{n \rightarrow \infty}\left|c_{n}\left(f_{\epsilon}\right)\right|^{1 / n}=M^{\prime}, M^{\prime} \leqq M$ $\leqq M^{\prime}+\epsilon$. By the second part of Theorem 4 , it follows that the series for $f_{\epsilon}$ diverges outside of $L_{M^{\prime}}$. But since the set of polynomials is total, the series must converge for all $B$. Therefore $B$ is contained in $L_{M^{\prime}}$. By letting $\epsilon \rightarrow 0$, it follows that $B$ must be contained in $L_{M}$.

4. Application to conformal mapping. We shall next apply Theorem 1 to some problems in the theory of conformal mapping. All the domains with which we shall be dealing in the present section will be assumed to have analytic boundaries. Let

$$
z=\psi(w)=w+a_{0}+\frac{a_{1}}{w}+\frac{a_{2}}{w^{2}}+\cdots,
$$

give a schlicht conformal map of the exterior of the circle $|w|=a, a>0$, onto the exterior of a domain $B$. The inverse map will be denoted by $\phi(z)$. The positive quantity $a$ is known as the transfinite diameter of $B$. Inasmuch as the boundary of $B$ is assumed to be analytic, the mapping function $\psi(w)$ may be continued across the circle $|w|=a$. There will therefore exist an $r: 0<r<a$, such that the mapping function $\psi(w)$ will continue to be regular and schlicht in the exterior of $|w|=r$. We shall derive a lower bound for such values $r$, and in the case of the interior mapping function, an analogous upper bound. These bounds will be given in terms of simple geometric quantities associated with the domain $B$.

We begin with a proof of an asymptotic expression for the Faber polynomials (9) of a domain $B$ (cf. Szegö [10, p. 363]).

THEOREM 6. Let $w=\phi(z)$ map the exterior of $B$ conformally onto the exterior of $|w|=a$. If $z=\psi(w)$ is regular and schlicht for $|w| \geqq r, r<a$, and if $C_{r}$ designates the image in the $z$-plane of the circle $|w|=r$, then we have

$$
\lim _{n \rightarrow \infty} p_{n}(z) /[\phi(z)]^{n}=1
$$


holding uniformly for all $\mathrm{z}$ exterior to $C_{r}$.

Proof. Let $|z|=R$ be a large circle. We consider the integral

$$
\frac{1}{2 \pi i} \int_{|\zeta|=R} \frac{[\phi(\zeta)]^{n}-p_{n}(\zeta)}{\zeta-z} d \zeta
$$

The integrand is analytic for $|\zeta| \geqq R$ (see (9)), and at the point $\zeta=\infty$ has a zero of order at least two, inasmuch as $[\phi(\zeta)]^{n}-p_{n}(\zeta)$ has a zero of order at least one. The residue at $\infty$ of (32) is therefore 0 , so that

$$
\frac{1}{2 \pi i} \int_{|\zeta|=R} \frac{[\phi(\zeta)]^{n}}{\zeta-z} d \zeta=\frac{1}{2 \pi i} \int_{|\zeta|=R} \frac{p_{n}(\zeta)}{\zeta-z} d \zeta=p_{n}(z) .
$$

Let $z$ be exterior to $C_{r}$. Since $\psi(w)$ is regular and schlicht for $|w| \geqq r$, there exists an $r^{\prime}<r$ for which the same may be asserted. Now

$$
[\phi(z)]^{n}=\frac{1}{2 \pi i} \int_{|\zeta|=R} \frac{[\phi(\zeta)]^{n}}{\zeta-z} d \zeta-\frac{1}{2 \pi i} \int_{C_{r^{\prime}}} \frac{[\phi(\zeta)]^{n}}{\zeta-z} d \zeta,
$$

so that

$$
p_{n}(z)=[\phi(z)]^{n}+\frac{1}{2 \pi i} \int_{C_{r^{\prime}}} \frac{[\phi(\zeta)]^{n}}{\zeta-z} d \zeta,
$$

whence

$$
\left|p_{n}(z)-[\phi(z)]^{n}\right| \leqq \frac{L\left(C_{r^{\prime}}\right) r^{\prime n}}{2 \pi \operatorname{dist}\left(z, C_{r^{\prime}}\right)} .
$$

Thus,

$$
\left|\frac{p_{n}(z)}{[\phi(z)]^{n}}-1\right| \leqq \frac{L\left(C_{r^{\prime}}\right)}{2 \pi \operatorname{dist}\left(z, C_{r^{\prime}}\right)}\left(r^{\prime} / r\right)^{n},
$$

for all $z$ on and exterior to $C_{r}$. (31) now follows by letting $n \rightarrow \infty$.

The following corollary is an easy consequence of (37).

COROLlaRY 6.1. The zeros of the Faber polynomials are uniformly bounded. Their limit points are all confined to the interior of $C_{r}$.

Theorem 7. Let the domain $B$ have an analytic boundary $C$. Let $C_{r}$ be defined as in the previous theorem. Then the lines $l \in N(C)$ must all have points in common with $C_{r}$.

Proof. For if not, there would be an $l_{1} \in N(C)$ with $C_{r}$ lying completely to one side of it. By the preceding corollary, all the limit points of the zeros of the Faber polynomials for $B$ would therefore lie on one side of $l_{1}$. But this contradicts Theorem 1. 
Before proving our next theorem, it will be convenient to introduce a domain constant as follows: $r_{e}$ will be defined as the greatest lower bound of values $r$ for which the exterior mapping function $\psi(w)$ is regular and schlicht exterior to $|w|=r$. In addition, we shall introduce the function

$$
K(x)=\left(x-\left(x^{2}-x\right)^{1 / 2}\right)^{-2} ; \quad x \geqq 1 .
$$

It is to be noted that the function $K(x)$ increases monotonically from $K(1)=1$ to $K(\infty)=4$.

THEOREM 8. Let the boundary of $B$ be an analytic curve $C$. If

$$
d^{*}=\min _{z \in \bar{B}} \max _{l \in N(C)} \operatorname{dist}(z, l),
$$

then we have

$$
d^{*} / 4 \leqq r_{0}<a .
$$

More precisely, if

$$
m=\min \operatorname{dist}(0, \text { bdry } B),
$$

then we have

$$
d^{*} \leqq r_{e} K\left(r_{e} / m\right) \text {. }
$$

Proof. The right-hand inequality of (40) is immediate, inasmuch as $C$ is analytic. Let the mapping function $\psi(w)$ be regular and schlicht on and exterior to the circle $|w|=r$. At the outset, we shall make the further assumption that $\psi(w)$ does not vanish in this region. In this case, the image $C_{r}$ of $|w|=r$ will be a curve which contains the origin in its interior. Consider now the function

$$
h(w)=r / \psi(r / w)=w+\cdots .
$$

The function $h(w)$ is regular and schlicht in $|w| \leqq 1$, mapping it on the interior of a curve $C^{I}$ which is obtained by inverting $C$ in the circle $|z|=r$. If $M^{I}=\max$ dist $\left(0, C^{I}\right)$, then $M^{I}=r / m$. By the distortion theorem of Pick [8], we shall have

$$
\min _{|w|=1}|h(w)| \geqq 1 / K(r / m)
$$

and hence

$$
\max _{|w|=r}|\psi(w)| \leqq r K(r / m) .
$$

If, now, $d=\max _{l \in N(C)}$ dist $(0, l)$, then by the same argument used in the proof of Theorem 7 , we must have

$$
d \leqq r K(r / m)<4 r .
$$


In the general situation, i.e., if $\psi(w)$ vanishes in $|w| \geqq r$, then a constant $c$ may be chosen so that the function $\psi^{\prime}(w)=\psi(w)+c$ will map the exterior of $|w|=a$ onto the exterior of the congruent domain $B^{\prime}$ so that the origin $0^{\prime}$ is located interior to the curve $C_{r}^{\prime}$. In this case, (46) will hold with $d$ replaced by $d^{\prime}=\max _{l \in N(C)}$ dist $\left(0^{\prime}, l\right)$. But now from (39), we have $d^{*} \leqq d^{\prime}$, so that $d^{*} \leqq r K(r / m)<4 r$. The inequalities (40) and (42) now follow.

Let us describe some of the consequences of this theorem. The transfinite diameter $a$ is an increasing set function, so that if $R$ is the radius of the smallest circle containing $B$, we must have $a \leqq R$. (40) may therefore be rewritten in the weakened form

$$
d^{*} / 4 R \leqq r_{e} / a .
$$

Consider now domains $B$ lying interior to the unit circle (i.e., $R=1$ ). If the exterior mapping function $\psi(w)$ can be continued into the far interior of the circle $|w|=a$, then $r_{e} / a$ and hence $d^{*}$ will be very small. This implies that the domain $B$ must be "circle-like." (In this connection, we remark that $d^{*}=0$ if and only if $B$ is a circle.) On the other hand, if $C$ bounds an area which is long and thin, or consists of a circle-like figure with a small bump in it, then, as can be seen from Theorem 7 , the maximum deviation of curves $C_{r}, r<a$, from $C$ must be small. For such figures, the inverse mapping function $\phi(z)$ cannot be continued to any great extent into the interior of $B$ so as to cover circles $|w|=r$.

We shall now obtain theorems similar to Theorems 7 and 8 for the interior mapping function $z=f(w)=w+a_{2} w^{2}+\cdots$. They are obtained from the two preceding theorems by inversion in the unit circle.

THEOREM 9. Let the boundary of $B$ be an analytic curve $C$, and suppose that $z=f(w)=w+a_{2} w^{2}+\cdots$ maps the interior of $|w|=b$ onto the interior of $B$. If $f(w)$ can be continued so as to be regular and schlicht in $|w| \leqq r, r>b$, then the curve $C_{r}: f\left(r e^{i \theta}\right), 0 \leqq \theta \leqq 2 \pi$, must meet all the circles which pass through $z=0$ and are orthogonal to $C$.

Proof. Consider the function $h(w)=1 / f(1 / w)=w+\cdots$. The function $h(w)$ is regular and schlicht on and exterior to $|w|=1 / r$, mapping it onto the exterior of a curve $C_{r}^{I}$, the point at infinity going into the point at infinity. $C_{r}^{I}$ is the inverse of $C_{r}$ in $|z|=1$. By Theorem 7 , any normal to $C^{I}$ must meet $C_{\tau}^{I}$. Inverting this figure in the unit circle, a normal to $C^{I}$ goes into a circle passing through $z=0$ and orthogonal to $C$, and vice versa, and hence these circles must meet $C_{r}$.

In the statement of Theorem 9, we have used only the images of normals to $C^{I}$. The theorem holds for the images of all lines $l \in N\left(C^{I}\right)$. These images constitute a family of circles $O(C)$ which may be obtained as follows. Let $z_{1}$ and $z_{2}$ be two arbitrary. points lying on $C$. Let $c\left(0, z_{1}, z_{2}\right)$ designate the circle passing through the indicated points. The harmonic mean of $z_{1}$ and $z_{2}$, h.m. $\left(z_{1}, z_{2}\right)$ 
$=\left\{\left(z_{1}^{-1}+z_{2}^{-1}\right) / 2\right\}^{-1}$, must also lie on $c\left(0, z_{1}, z_{2}\right)$. Construct the circle passing through the origin and h.m. $\left(z_{1}, z_{2}\right)$ and orthogonal at the latter point to $c\left(0, z_{1}, z_{2}\right) . O(C)$ is the family of all such circles $\left({ }^{2}\right)$.

Theorem 9 may be worded in the following slightly altered form: Let $z=f(w)=w+\cdots$ be regular and schlicht in $|w| \leqq r$. Then all the circles of the family $O\left(C_{r_{1}}\right), r_{1}<r$, must have points in common with $C_{r}$. This has an interesting geometric consequence, as $r_{1} \rightarrow 0$. At the point $P$ on $C_{r_{1}}$, let $\alpha$ denote the smaller angle between the normal to $C_{r_{1}}$ and the radius vector $O P$. This angle measures the departure from circularity of $C_{r_{1}}$ at $P$. If the circle $\Delta$ passing through $O$ and $P$ and orthogonal at $P$ to $C_{r_{1}}$ has the diameter $D$ $=D(P)$, then it is easily seen that

$$
\sin \alpha=O P / D=|f| / D \text {. }
$$

Now, as $r_{1} \rightarrow 0,|f| \rightarrow 0$, while by Theorem $9, D \geqq m>0$. Hence $\alpha \rightarrow 0$. Thus, the images of circles $|w|=r_{1}, r_{1}$ small, must be increasingly circle-like. In order to make this statement more precise, as well as to show that distortion theorems for schlicht functions of a type investigated by Golusin [5] may be derived from Theorem 9, we shall next prove a lemma which expresses the diameter $D$ directly in terms of the mapping function $f(w)$.

Lemma. Let $z=f(w), f(0)=0$, be regular in $|w| \leqq r$, mapping the circumference onto $C_{r}$. Let $P=P\left(r, \theta_{0}\right)$ be the image of $w_{0}=r e^{i \theta_{0}}$. Then the circle $\Delta$ passing through 0 and $P$ and orthogonal to $C_{r}$ has diameter $D$ given by

$$
D=|f| / \sin \left|\arg w f^{\prime}(w) / f(w)\right|,
$$$$
w=w_{0} .
$$

Proof. We have $\partial f / \partial \theta=i w f^{\prime}(w)$. The slope of $C_{r}$ at $P$ is $\tan \arg \partial f\left(r e^{i \theta}\right) /\left.\partial \theta\right|_{\theta_{0}}$. Hence, the slope of the normal is - ctn arg $\partial f /\left.\partial \theta\right|_{\theta_{0}}=-\left.\operatorname{ctn} \arg i w f^{\prime}(w)\right|_{w_{0}}$ $=\left.\tan \arg w f^{\prime}(w)\right|_{w_{0}}$. The slope of the radius vector $0 P$ is $\tan \arg f\left(w_{0}\right)$. Hence, if $\alpha^{*}, 0 \leqq \alpha^{*}<\pi$, designates the angle from the normal to $0 P$, we have $\tan \arg w f^{\prime} /\left.f\right|_{w_{0}}=\tan \alpha^{*}$. Therefore,

$$
\begin{aligned}
& \alpha^{*}=\arg w f^{\prime} /\left.f\right|_{w_{0}} ; 0 \leqq \arg w f^{\prime} /\left.f\right|_{w_{0}}<\pi, \\
& \alpha^{*}=\arg w f^{\prime} /\left.f\right|_{w_{0}}+\pi ; \quad-\pi \leqq \arg w f^{\prime} /\left.f\right|_{w_{0}}<0 .
\end{aligned}
$$

In either case, $\left|\arg w f^{\prime} / f\right|_{w_{0}}$ is one of the angles between the normal and the radius vector. Formula (49) now follows from (48).

THEOREM 10. Let $z=f(w)=w+a_{2} w^{2}+\cdots$ be regular and schlicht in $|w| \leqq 1$, mapping this circle onto a domain $B$. Let

$$
M=\max \operatorname{dist}(0, \text { bdry } B) \text {. }
$$

Then,

- (2) It is easily seen that $O(C)$ can also be described as the family of all circles $A\left(z_{1}, z_{2}, t\right)$, $t<1, z_{1}, z_{2} \in C$, which pass through $z=0$. 


$$
\arg \left|w f^{\prime}(w) / f(w)\right| \leqq \arcsin [K(M)|f|],
$$

whenever the right-hand member is defined.

Proof. If $m$ is defined as in (41), then by the distortion theorem of Pick, $m \geqq 1 / K(M)$. By Theorem 9 , all circles $\Delta$ must have points in common with the boundary of $B$. Therefore, by our lemma,

$$
|f| / \sin \left|\arg w f^{\prime}(w) / f(w)\right| \geqq m \geqq 1 / K(M),
$$

whence

$$
\begin{aligned}
& \left|\arg w f^{\prime}(w) / f(w)\right| \leqq \arcsin [K(M)|f|], \\
& \left|\arg w f^{\prime}(w) / f(w)\right| \leqq \arcsin [4|f|] .
\end{aligned}
$$

COROLlaRY 10.1(3). Let $\alpha=\alpha(P)$ designate the smaller angle between the normal to $C_{r}$ at $P$ and the radius vector $0 P$. Then,

$$
\lim _{r \rightarrow 0} \sup \alpha / r \leqq K(M) .
$$

Proof. By the Koebe Verzerrungsatz, $|f| \leqq r /(1-r)^{2}$. This estimate, combined with (54a), yields the stated result.

We remark that in Golusin [5] it is shown that if $f(w)=w+a_{2} w^{2}+\cdots$ is regular and schlicht in $|w|<1$, then

$$
\left|\arg w f^{\prime}(w) / f(w)\right| \leqq \log \frac{1+|w|}{1-|w|} .
$$

The lim sup in (55) may therefore be improved as follows

$$
\limsup _{r \rightarrow 0} \alpha / r \leqq \min (K(M), 2) .
$$

The estimates (54) are essentially independent of (56). For, referring to $(54 \mathrm{~b})$, select $f(w)=w /(1-w)^{2}$, and $w_{0}=-\tanh \pi / 4=-.65$. Then, $\log \left(1+\left|w_{0}\right|\right) /\left(1-\left|w_{0}\right|\right)=\pi / 2$, while $\arcsin \left|4 w_{0} /\left(1-w_{0}\right)^{2}\right|=\arcsin \left(1-e^{-\pi}\right)$ $<\pi / 2$.

For a given domain $B$, define a domain constant $r_{i}$ as the least upper bound of values $r$ such that the interior mapping function $f(w)=w+a_{2} w^{2}$ $+\cdots$ is regular and schlicht in $|w| \leqq r$.

THEOREM 11. Let $\delta$ equal the minimum diameter of circles passing through 0 and orthogonal to the boundary of $B$. Then,

$$
r_{i}<4 \delta \text {, }
$$

with the sharper inequality

$$
\delta \geqq r_{i} / K\left(M / r_{i}\right) \text {. }
$$

(3) For distortion theorems using a different definition of circularity, see Walsh [13]. 
Proof. Consider $h(w)=r^{-1} f(r w)=w+\cdots$. The function $h(w)$ is regular and schlicht in $|w| \leqq 1$, mapping it onto a domain $B^{\prime}$ for which the maximum distance from the origin to the boundary is $M / r$. By Pick's distortion theorem,

$$
\min _{|w|=1}|h(w)| \geqq 1 / K(M / r) \text {. }
$$

Hence,

$$
\min _{|w|=r}|f(w)| \geqq r_{i} / K\left(M / r_{i}\right)>r_{i} / 4 .
$$

But, by Theorem 9 ,

$$
\min _{|w|=r}|f(w)| \leqq \delta .
$$

Theorems 7 and 9 may be reformulated analytically as follows.

THEOREM 7a. Let $f(w)$ be regular and schlicht in $|w| \geqq 1$. Then, to every $w$, $|w|>1$, there exists $a \theta, 0 \leqq \theta \leqq 2 \pi$, such that

$$
\frac{f(w)-f(e)}{w f^{\prime}(w)}
$$

is real. In addition, to every pair $w_{1}, w_{2},\left|w_{1}\right|=\left|w_{2}\right|>1$, there exists a $\theta$ such that

$$
\frac{f\left(w_{1}\right)+f\left(w_{2}\right)-2 f\left(e^{i \theta}\right)}{f\left(w_{1}\right)-f\left(w_{2}\right)}
$$

is purely imaginary.

THEOREM 9a. Let $f(w)$ be regular and schlicht in $|w| \leqq 1$, and let $f(0)=0$, $f^{\prime}(0)=1$. Then, to every $w,|w|<1$, there exists a $\theta, 0 \leqq \theta \leqq 2 \pi$, such that

$$
w f^{\prime}(w)\left(\frac{1}{f(w)}-\frac{1}{f\left(e^{i \theta}\right)}\right)
$$

is real. In addition, to every pair $w_{1}, w_{2},\left|w_{1}\right|=\left|w_{2}\right|<1$, there exists a $\theta$ such that

$$
\frac{\frac{1}{f\left(w_{1}\right)}+\frac{1}{f\left(w_{2}\right)}-\frac{2}{f\left(e^{i \theta}\right)}}{\frac{1}{f\left(w_{1}\right)}-\frac{1}{f\left(w_{2}\right)}}
$$

is purely imaginary. 
5. The case of multiply-connected regions. In the present section, we shall obtain, for multiply-connected regions, theorems which are direct generalizations of Theorem 7 and 9. Instead of the Faber polynomials, we shall utilize certain sets of rational functions introduced by Walsh. In addition, we shall derive a theorem for total sets of rational functions corresponding to Theorem 1.

Let there be given a bounded region $B$ and a set $L_{B}$ of functions which are regular in $B$ and such that to each boundary point $z_{b}$ of $B$ and to each $\epsilon>0$, there exists a function $f \in L_{B}$ with a singularity in $C\left(z_{b} ; \epsilon\right)$. We shall say that the set of rationals

$$
r_{n}(z)=\frac{p_{n}(z)}{q_{n}(z)}=\frac{\left(z-\beta_{1}^{(n)}\right) \cdots\left(z-\beta_{n}^{(n)}\right)}{\left(z-\alpha_{1}^{(n)}\right) \cdots\left(z-\alpha_{n}^{(n)}\right)} \quad(n=1,2, \cdots)
$$

is total over $B$ if every function $f \in L_{B}$ has an expansion of the form

$$
f(z)=\sum_{n=1}^{\infty} a_{n} r_{n}(z)
$$

convergent at each point of $B$.

THEOREM 12. Let $z_{b_{1}}$ and $z_{b_{2}}$ be two arbitrary but distinct boundary points of $B$. If a set of rationals $r_{n}(z)$ is total over $B$, and if all the zeros $\beta_{i}^{(n)}$ lie in the circle $A=A\left(z_{b_{1}}, z_{b_{2}} ; t\right), t<1$, then there must be a limit point of poles $\alpha_{i}^{(n)}$ in $A$.

Proof. Let us note at the outset that for $t>1$, the regions $A\left(z_{1}, z_{2} ; t\right)$ where $z$ satisfies $\left|\left(z-z_{1}\right) /\left(z-z_{2}\right)\right| \leqq t$ are no longer bounded but are determined by the relation $A\left(z_{1}, z_{2} ; t\right)=\left(\operatorname{comp} A\left(z_{2}, z_{1} ; 1 / t\right)\right)^{-}$. Let $z_{I}$ denote an arbitrary but fixed point interior to $B$. We shall prove initially that it is impossible to have

$$
\begin{aligned}
& \beta_{i}^{(n)} \in A\left(z_{b_{1}}, z_{I} ; t_{1}\right), \\
& \alpha_{i}^{(n)} \in A\left(z_{I}, z_{b_{1}} ; t_{2}\right) \quad\left(i=1,2, \cdots, n ; n \geqq n_{0} ; t_{1} t_{2}<1\right) .
\end{aligned}
$$

Select an $\epsilon$ so small that $C\left(z_{b_{1}} ; \epsilon\right) \subset A\left(z_{b_{1}}, z_{I} ; t_{1}\right)$, and assume that (62) holds with some fixed $t_{1}, t_{2}, t_{1} t_{2}<1$. We shall now estimate the product

$$
\pi_{i}^{(n)}=\frac{\left|z-\beta_{i}^{(n)}\right|}{\left|z_{I}-\beta_{i}^{(n)}\right|} \frac{\left|z_{I}-\alpha_{i}^{(n)}\right|}{\left|z-\alpha_{i}^{(n)}\right|}
$$

for $z \in C\left(z_{b_{1}} ; \epsilon\right)$. Let $d=\left|z_{b_{1}}-z_{I}\right|$, then

$$
\frac{\left|z-\beta_{i}^{(n)}\right|}{\left|z_{I}-\beta_{i}^{(n)}\right|} \leqq \frac{\left|z-z_{b_{1}}\right|+\left|z_{b_{1}}-\beta_{i}^{(n)}\right|}{\left|z_{I}-\beta_{i}^{(n)}\right|} \leqq \frac{\epsilon}{d / 2}+t_{1}
$$


while

$$
\begin{aligned}
\frac{\left|z_{I}-\alpha_{i}^{(n)}\right|}{\left|z-\alpha_{i}^{(n)}\right|} & =\frac{\left|z_{I}-\alpha_{i}^{(n)}\right|}{\left|z_{b_{1}}-\alpha_{i}^{(n)}-z_{b_{1}}+z\right|} \leqq \frac{\left|z_{I}-\alpha_{i}^{(n)}\right|}{\left|z_{b_{1}}-\alpha_{i}^{(n)}\right|-\epsilon} \\
& =\frac{\left|z_{I}-\alpha_{i}^{(n)}\right| /\left|z_{b_{1}}-\alpha_{i}^{(n)}\right|}{1-\frac{\epsilon}{\left|z_{b_{1}}-\alpha_{i}^{(n)}\right|}} \leqq \frac{t_{2}}{1-\frac{\epsilon}{(d / 2)-\epsilon}} .
\end{aligned}
$$

Therefore, by choosing $\epsilon$ sufficiently small, and in view of $t_{1} t_{2}<1$, we may make $\pi_{i}^{(n)} \leqq t^{\prime}<1\left(n \geqq n_{0} ; i=1,2, \cdots, n\right)$, for all $z$ in $C\left(z_{b_{1}} ; \epsilon\right)$. Now, let $f \in L_{B}$ possess a singularity in $C\left(z_{b_{1}} ; \epsilon\right)$. The function $f$ possesses an expansion (61) convergent in $B$, and hence, in particular, in the point $z_{I}$. Therefore we have, for some $M>0,\left|a_{n} p_{n}\left(z_{I}\right) / q_{n}\left(z_{I}\right)\right|<M(n=0,1, \cdots)$ so that $\left|a_{n}\right|$ $<M\left|q_{n}\left(z_{I}\right) / p_{n}\left(z_{I}\right)\right|$. The series (61) will therefore be dominated by

$$
M \sum_{n=1}^{\infty}\left|\frac{q_{n}\left(z_{I}\right)}{p_{n}\left(z_{I}\right)} \frac{p_{n}(z)}{q_{n}(z)}\right|<M^{\prime} \sum_{n=1}^{\infty} t^{\prime n}<\infty
$$

for $z \in C\left(z_{b_{1}} ; \epsilon\right)$. The series (61) will therefore converge uniformly and absolutely in $C\left(z_{b_{1}} ; \epsilon\right)$ and thus represents an analytic continuation of $f(z)$ to $C\left(z_{b_{1}} ; \epsilon\right)$. This is impossible.

Suppose now that all the zeros are located in $A\left(z_{b_{1}} ; z_{I} ; t_{1}\right), t_{1}<1$. Set $t_{2}=t_{1}+\epsilon$. For $\epsilon$ sufficiently small, $\left(t_{1}+\epsilon\right)^{-1}>1$. By the previous result, it is impossible that all but a finite number of poles lie in $A\left(z_{I}, z_{b_{1}} ;\left(t_{1}+\epsilon\right)^{-1}\right)$ $=$ comp int $A\left(z_{b_{1}}, z_{I}, t_{1}+\epsilon\right)$. Therefore there must be a limit point of poles in $A\left(z_{b_{1}}, z_{I}, t_{1}+\epsilon\right)$. By letting $\epsilon \rightarrow 0$, we can therefore conclude that if all the zeros are located in $A\left(z_{b_{1}}, z_{I}, t\right), t<1$, then there must be a limit point of poles in the same circle.

Finally, let $z_{I_{n}}$ be a sequence of interior points such that $\lim _{n \rightarrow \infty} z_{I_{n}}=z_{b_{2}}$. To every $\epsilon>0$, there will be an $n_{0}$ such that the circles $A\left(z_{b_{1}}, z_{I_{n}} ; t+\epsilon\right)\left(n \geqq n_{0}\right)$ all contain the circle $A\left(z_{b_{1}}, z_{b_{2}} ; t\right)$ in their interior. If, therefore, all the zeros are assumed to lie in the latter, then there must be a limit point of poles in $A\left(z_{b_{1}}, z_{I_{n}} ; t+\epsilon\right)$. The result now follows by letting $\epsilon \rightarrow 0, n \rightarrow \infty$.

We now digress briefly to describe the process of interpolation by rational functions (cf. Walsh [12]). A rational function

$$
r_{n}(z)=\frac{b_{n 0} z^{n}+b_{n 1} z^{n-1}+\cdots+b_{n n}}{\left(z-\alpha_{1}^{(n)}\right) \cdots\left(z-\alpha_{n}^{(n)}\right)}
$$

is said to interpolate to the function $f(z)$ at the points $\beta_{i}^{(n)}(i=1,2, \cdots, n)$ if $f\left(\beta_{i}^{(n)}\right)=r_{n}\left(\beta_{i}^{(n)}\right)$. The fundamental problem of interpolation by rationals is to study the convergence of $r_{n}(z)$ thus determined to $f(z)$. In the particular case 
where the zeros and the poles do not depend on $n$, i.e., $\beta_{k}^{(n)}=\beta_{k}, \alpha_{k}^{(n)}=\alpha_{k}$, it may be shown that the $r_{n}(z)$ which interpolate to $f(z)$ may be expressed in the form

$$
r_{n}(z)=\sum_{j=1}^{n} L_{j}(f) \frac{\left(z-\beta_{1}\right) \cdots\left(z-\beta_{j}\right)}{\left(z-\alpha_{1}\right) \cdots\left(z-\alpha_{j}\right)}
$$

where $L_{j}$ indicates a certain fixed (i.e., independent of $n$ and $f$ ) linear functional. In this case, the convergence of $r_{n}(z)$ to $f(z)$ is equivalent to the statement

$$
f(z)=\sum_{j=1}^{\infty} L_{j}(f) \frac{\left(z-\beta_{1}\right) \cdots\left(z-\beta_{j}\right)}{\left(z-\alpha_{1}\right) \cdots\left(z-\alpha_{j}\right)} .
$$

For the sake of convenience of reference, we now state two theorems on interpolation by rationals which are due to Walsh. Our statement will be found to be less general than that given in [12].

THEOREM 13a. Let $B$ be a bounded region whose boundary $b$ consists of $a$ finite number of mutually disjoint analytic Jordan curves, and let the origin $z=0$ lie in $B$. Then there exists a sequence of points $\alpha_{1}, \alpha_{2}, \cdots$ on $b$ with the following property. Let $G(z, 0, B)$ designate the Green's function of $B$ with singularity $\log 1 /|z|$. If the function $f(z)$ is regular in $G_{r}: G(z, 0, B)>r \geqq 0$, then it possesses an expansion of the form

$$
f(z)=\sum_{n=0}^{\infty} a_{n} \frac{z^{n}}{\left(z-\alpha_{1}\right) \cdots\left(z-\alpha_{n}\right)}
$$

valid in $G_{r}$.

THEOREM 13b. Let $C_{0}$ and $C_{1}$ be two nonintersecting analytic Jordan curves or nonintersecting sets each of a finite number of nonintersecting curves. Suppose that a region $R$ is bounded by $C_{0}$ and $C_{1}$ and that one curve of the set $C_{0}$ contains all the other curves of the set $C_{0}$ and all the curves of the set $C_{1}$. Let $u(z)$ be harmonic in $R$, continuous in $\bar{R}$, and such that $u(z)=0, z \in C_{0} ; u(z)=1, z \in C_{1}$. Let $R_{\mu}$ be the region or set of regions bounded by the complete locus $u(z)=\mu$, $0<\mu<1, R_{\mu}$ containing in its interior no point of $C_{0}$. Then there exist sets of points $\alpha_{k}$ and $\beta_{k}$ lying on $C_{0}$ and $C_{1}$ respectively, such that if $f(z)$ is analytic in $\bar{R}_{\mu}$, then (61) holds uniformly in $R_{\mu}$.

The set of rationals appearing in (68) and the set determined as above are therefore total over their respective regions of expandability. We now apply Theorem 12 to each of these theorems.

THEOREM 14. Let the region $B$ be bounded by a finite number of mutually disjoint analytic Jordan curves $b$, and let the origin $z=0$ lie in $B$. Suppose that the Green's function $G(z, 0, B)$ can be continued across all boundaries to the value 
$r^{\prime}, r^{\prime}<0$, and that $b^{\prime}$ designates the curve or sets of curves satisfying $G(z, 0, B)$ $=r^{\prime}$, it being assumed that one curve of $b^{\prime}$ contains the whole configuration. Then $b^{\prime}$ must intersect each member of the family of circles $O(b)$. (See pp. 9495 for the definition of this family.)

Proof. Designate by $B^{\prime}$ the region bounded by the set of curves $b^{\prime}$. We have $G(z, 0, B)-r^{\prime}=G\left(z, 0, B^{\prime}\right)$ and therefore the curves of $b$ are the level lines $G\left(z, 0, B^{\prime}\right)=-r^{\prime}$. By Theorem 13a, there exists a set of rationals $z^{n} /\left(z-\alpha_{1}\right) \cdots\left(z-\alpha_{n}\right), \alpha_{i} \in b^{\prime}$, which are total over $B$. Each member of the family $O(b)$ by definition passes through $z=0$, and therefore contains all the zeros of this set of rationals. Hence by Theorem 12, each circle of this family must contain a limit point of poles. But these are located on the curves $b^{\prime}$, and the theorem therefore follows.

The following observations should be made at this point. Let the components of $b$ be designated by $b_{1}, b_{2}, \cdots, b_{n}$ and those of $b^{\prime}$ by $b_{1}^{\prime}, b_{2}^{\prime}, \cdots, b_{n}^{\prime}$. If any circle of the family $O(b)$ fails to have points in common with $b_{1}, b_{2}, \cdots$, $b_{i-1}, b_{i+1}, \cdots, b_{n}$, then it surely must have points in common with $b_{i}^{\prime}$. If the region $B$ is simply-connected, then the curve $b^{\prime}$ must have points in common with each member of $O(b)$. Since for this case, the level lines of the Green's function are the radienbilder of the mapping function, we have again established, using the Walsh rationals, Theorem 9 in the general form. Thus Theorem 14 is the direct generalization of Theorem 9 to the case of multiplyconnected regions.

TheOREM 15. Let $C_{0}$ and $C_{1}$ be as in Theorem $13 \mathrm{~b}$, the curve $C_{0}^{(1)}$ containing the whole configuration. Let also $u(z)$ be the harmonic function described in Theorem $13 \mathrm{~b}$, and let $C_{-\eta}$ designate the analytic Jordan curve or curves which are the level lines of $u(z)$ assumed to be continued across $C_{0}: u(z)=-\eta, z \in C_{-\eta}$, $\eta>0$. Suppose furthermore that one curve $C_{-\eta}^{(1)}$ contains the whole configuration. If, now, $z_{b_{1}}$ and $z_{b_{2}}$ are two arbitrary boundary points of $C_{0}$ and if $A\left(z_{b_{1}}, z_{b_{2}} ; t\right)$, $t<1$, contains $C_{1}$, then it must have points in common with $C_{-\eta}^{(1)}$.

Proof. Let $u^{*}(z)=(u(z)+\eta) /(1+\eta)$. Then $u^{*}$ is harmonic, is 0 on $C_{-\eta}$, and 1 on $C_{1}$. The level lines $u^{*}(z)=\eta /(1+\eta)$ are precisely $C_{0}$. Thus, by Theorem $13 \mathrm{~b}$, there exist points $\beta_{i}$ on $C_{1}$ and $\alpha_{i}$ on $C_{-\eta}$ such that the set of rationals (67) is total over the region $R_{\mu}, \mu=\eta /(1+\eta)$. If, now, all the curves of $C_{1}$ are assumed to lie in a circle $A\left(z_{b_{1}}, z_{b_{2}} ; t\right), t<1, z_{b_{1}} \in C_{0}$, then a fortiori all the points $\beta_{i}$ lie in this circle. The conclusion now follows by an application of Theorem 12.

It is to be observed that Theorem 15 gives information as to the possible continuation of $u(z)$ only in the case where the original configuration $C_{0}, C_{1}$ is such that $C_{1}$ is contained in a circle $A\left(z_{b_{1}}, z_{b_{2}} ; t\right), t<1, z_{b_{i}} \in C_{0}$.

A similar theorem may be derived for the continuation of $u(z)$ across the curves $C_{1}$. Generalization to the case where some of the boundary curves are not analytic is also possible. For the case of doubly-connected regions, 
Theorem 15 is immediately translatable into a distortion theorem for functions which are schlicht in an annulus.

THEOREM 16. Let $w=f(z)$ be regular and schlicht in the annulus $0<r_{0} \leqq|z|$ $\leqq r_{1}<\infty$. If $r_{0}<r^{*}<r_{1}$, and if, for some $t<1$, we have

$$
\left|\frac{f\left(r_{0} e^{i \theta}\right)-f\left(r^{*} e^{i \theta_{1}}\right)}{f\left(r_{0} e^{i \theta}\right)-f\left(r^{*} e^{i \theta_{2}}\right)}\right| \leqq t
$$

for $0 \leqq \theta \leqq 2 \pi, \theta_{1}, \theta_{2}$ arbitrary, then there must exist a $\theta_{3}$ such that

$$
\left|\frac{f\left(r_{1} e^{i \theta_{3}}\right)-f\left(r^{*} e^{i \theta_{1}}\right)}{f\left(r_{1} e^{i \theta_{3}}\right)-f\left(r^{*} e^{i \theta_{2}}\right)}\right| \leqq t .
$$

Proof. Designate the images $f\left(r_{i} e^{i \theta}\right), 0 \leqq \theta \leqq 2 \pi$, by $C_{i}(i=0,1)$. Let $u(w)$ be that function which is harmonic in the region bounded by $C_{0}$ and $C_{1}$, and is 0 and 1 on $C_{0}$ and $C_{1}$ respectively. Set $u^{*}(w)=\log \left(r_{0} / r_{1}\right) u(w)+\log r_{1}$, and let $v^{*}(w)$ be its harmonic conjugate. This is not single-valued, but $f^{-1}(w)$ $=\exp \left[u^{*}(w)+i v^{*}(w)\right]$ is single-valued. It is now clear that the level lines of $u^{*}$, and hence of $u$, are the images of the concentric circles $0<r_{0} \leqq r \leqq r_{1}<\infty$. The result now follows from an application of Theorem 15.

Roughly put, we may say that if there be given two analytic curves $C_{0}, C_{1}$ with $C_{0}$ containing $C_{1}$ and the latter located sufficiently "off center" (so that (69) is satisfied), then the level lines of $u$ which surround $C_{0}$ must all lie close to $C_{0}$. Indeed they must all pass through each circle $A\left(z_{b_{1}}, z_{b_{2}} ; t\right)$. This result may also be interpreted as a minimum modulus theorem for functions which are schlicht in an annulus.

\section{BIBLIOGRAPHY}

1. S. Bergman, Über die Entwicklung der Harmonischen Funktionen der Ebene und des Raumes nach Orthogonalfunktionen, Math. Ann. vol. 86 (1922) pp. 237-271.

2. R. P. Boas, Jr., Basic sets of polynomials, I, Duke Math. J. vol. 15 (1948) pp. 717-724.

3. ' $T$. Carleman, Über die Approximation analytischer Funktionen durch lineare Aggregate von vorgegebenen Potenzen, Arkiv för Matematik, Astronomi, och Fysik vol. 17 (1922).

4. G. Faber, Über polynomische Entwicklungen, Math. Ann. vol. 57 (1903).

5. G. M. Golusin, Interior problems of the theory of schlicht functions, Trans. from the Russian, Office of Naval Research, Washington, 1947.

6. V. L. Goncharov, Teoriya Interpolirovaniya i Priblizheniyz Funktzii, Moscow-Leningrad, 1934.

7. P. Korovkin, Sur une généralisation de la série de Taylor, C. R. (Doklady) Acad. Sci. URSS vol. 14 (1937).

8. G. Pick, Über die konforme Abbildung eines Kreises auf ein schlichtes und zugleich beschränktes Gebiet, Sitzungsberichte der k. Akademie d. Wissenschaften, Vienna, Abt IIa, vol. 126 (1917) pp. 247-263.

9. G. Szegö, Über orthogonale Polynome, die zu einer gegebenen Kurve der komplexen Ebene gehören, Math. Zeit. vol. 9 (1921).

10. - Orthogonal polynomials, Amer. Math. Soc. Colloquium Publications, vol. 23, New York, 1938. 
11. J. L. Walsh, Lemniscates and equipotential curves, Amer. Math. Monthly vol. 42 (1935).

12. - - Interpolation and approximation by rational functions in the complex domain, Amer. Math. Soc. Colloquium Publications, vol. 20, New York, 1935.

13. - On the shape of level curves of Green's function, Amer. Math. Monthly vol. 44 (1937) pp. 202-213.

14. J. M. Whittaker, Séries de base de polynomes quelconques, Collection Borel, Paris, 1949.

HARVARD UNIVERSITY,

Cambridge, Mass. 